\title{
Submillimeter-resolution radiography of shielded structures with laser-accelerated electron beams
}

\author{
Vidya Ramanathan, Sudeep Banerjee, Nathan Powers, Nathaniel Cunningham, \\ Nathan A. Chandler-Smith, Kun Zhao, Kevin Brown, and Donald Umstadter \\ Department of Physics and Astronomy, University of Nebraska, Lincoln, Nebraska 68588-0111, USA \\ Shaun Clarke and Sara Pozzi \\ Department of Nuclear Engineering \& Radiological Sciences, University of Michigan, Ann Arbor, Michigan 48109-2104, USA \\ James Beene, C R Vane, and David Schultz \\ Physics Division, Oak Ridge National Laboratory, Oak Ridge, Tennessee 37831-6372, USA
}

(Received 24 March 2010; published 14 October 2010)

\begin{abstract}
We investigate the use of energetic electron beams for high-resolution radiography of flaws embedded in thick solid objects. A bright, monoenergetic electron beam (with energy $>100 \mathrm{MeV}$ ) was generated by the process of laser-wakefield acceleration through the interaction of 50-TW, 30-fs laser pulses with a supersonic helium jet. The high energy, low divergence, and small source size of these beams make them ideal for high-resolution radiographic studies of cracks or voids embedded in dense materials that are placed at a large distance from the source. We report radiographic imaging of steel with submillimeter resolution.
\end{abstract}

DOI: 10.1103/PhysRevSTAB.13.104701

PACS numbers: 52.38.Kd, 52.59.-f

\section{INTRODUCTION}

Laser-plasma based electron accelerator technology has advanced considerably since it was first proposed by Tajima and Dawson [1]. The motivation behind this rapidly growing technology is the need for high quality particle beams that possess high spatial finesse along with monochromatic energy distribution, which is a requirement for most practical applications [2]. Laser-wakefield accelerators have been shown to produce electron beams with source sizes comparable to the laser beam waist [3]. Several groups now routinely generate monoenergetic electron beams of energy $100-300 \mathrm{MeV}$ that contain $>10-500-p C$ charge and an angular divergence of a few $\operatorname{mrad}$ [4-8]. The small foot-print $\left(\sim 100 \mathrm{ft}^{2}\right)$ of such lasers makes them potentially portable. These attributes make it worthwhile to consider the application of these energetic electron beams to nondestructive evaluation of defects in large structures, e.g., turbine blades and nuclear reactor vessels. These defects may range from a few microns to larger embedded cracks. In order for a radiography source to be useful for these applications, it must have special characteristics. Clearly, the source must be based on energetic beams in order to achieve the desired level of penetration in dense material. The radiation must be produced in a well-collimated beam to permit transport over large distances without loss of brightness. In order to spatially resolve microstructures, the intrinsic size of the source should be on the order of microns. From the standpoint of economic viability, the source and the associated detection system must be compact and relatively inexpensive. It must be possible to raster the source in a controlled way since any high-brightness beam would be significantly smaller in size than the object being interrogated. As proximity of the source to the object under investigation is not always a practical possibility, it is desirable for an imaging apparatus to be located at a large distance. Laserdriven electron beams possess all of these characteristics required for this unique and challenging interrogation modality.

In this paper, we investigate the fundamental and practical limits of the use of laser-wakefield driven high-energy electron beams for nondestructive radiographic imaging of submillimeter structures embedded within dense objects at a great distance from the beam source. When an energetic electron beam travels through matter, the Coulomb force between the electrons and the nuclei in the medium leads to a loss of energy, along with a large number of small deflections in the electron trajectories. Electrons that penetrate dense material undergo significantly more elastic and inelastic collisions than those that traverse through air or low density materials. As a result, the electrons lose energy as they propagate through matter, and their angular distribution becomes broader. The energy loss and angular spread depends on the total areal density traversed, and can be used to infer the characteristics of the medium through which the electrons propagated. The transmitted electrons can be efficiently detected on scintillator screens placed after the target material. A scintillating detector records the $2 \mathrm{D}$ variations in deposited beam dose, with larger dose deficits corresponding to high areal density 
regions within the target material. The highly penetrating nature of high-energy electron beams, coupled with their low divergence, enables efficient delivery to a distant target, making them suitable for long-standoff (imaging object is placed at a large distance from the source) applications.

Bremsstrahlung $\mathrm{x}$-ray sources and neutron beams have typically been used for interrogation when the target is in close proximity to the source $[9,10]$. Unlike bremsstrahlung based sources (which exhibit peak penetration of matter at $10 \mathrm{MeV}$ ), higher electron energy equates to deeper penetration through dense media. For instance, a $0.5 \mathrm{GeV}$ electron beam has a range of $6 \mathrm{~cm}$ in iron and $627 \mathrm{~m}$ in air [11]. Proton beams of energy $800 \mathrm{MeV}$ have successfully been used as a radiographic source in conjunction with a magnetic lens system to focus the protons on to the image plane after interaction with the target [12]. While proton beams of energy $200 \mathrm{MeV}$ and higher have more penetrating power as compared to electron beams of the same energy, the cost of a $200 \mathrm{MeV}$ proton accelerator far exceeds the price of the laser generated electron accelerator currently in use at UNL, besides such an accelerator may not be mobile. Hence, the compactness of the laserdriven wakefield electron source compared to a conventional linear accelerator or a proton accelerator makes it feasible to consider the development and deployment of a portable electron accelerator.

Prior work on radiography using electron beams from a laser-wakefield accelerator [13] were relatively low resolution $(\sim 1$ - $\mathrm{mm})$, because the electron beam had a quasiMaxwellian spectrum, predominantly containing low energy electrons. The highest resolution $(\sim 0.5-\mathrm{mm})$ radiographs previously obtained were recorded using bremsstrahlung generated $\gamma$ rays, produced when a laser generated electron beam impinged on a Tantalum converter [14], with a source size inferred to be $320 \mu \mathrm{m}$. In this case, the object was placed in vacuum in close proximity $(\sim 10 \mathrm{~cm})$ to the source conditions which are unsuitable for applications where the distance between the source and the object being interrogated is large. We demonstrate direct electron beam radiographs that were performed with quasimonochromatic high-energy beams, for which small angle scattering is reduced. We were able to visually resolve embedded structures as small as 250microns wide and 2.54-cm in length, with the object placed in air at a distance of several meters from the source. The calculated resolution is $400 \mu \mathrm{m}$ and is limited by the signal-to-noise ratio.

\section{EXPERIMENTAL SETUP}

Pulses from the University of Nebraska, Lincoln, Diocles Ti:sapphire laser system were focused to an intensity of $1.7 \times 10^{19} \mathrm{~W} / \mathrm{cm}^{2}$ [corresponding to a normalized laser vector potential $\left(a_{0}=e A / \mathrm{mc}^{2}\right)$ of 2.8] in order to drive a relativistic plasma wave. The quasimonoenergetic electron beams that are routinely obtained are tunable over an energy range of 50-400 MeV, depending on the choice of acceleration distance (i.e. the nozzle diameter), plasma density, and laser intensity. For the purpose of radiography, $1.3 \mathrm{~J}$ of laser energy was focused over a $3 \mathrm{~mm}$ diameter gas jet, of the Laval type, with initial plasma density of $1 \times 10^{19} \mathrm{~cm}^{-3}$. The experimental setup used is shown in Fig. 1. A permanent rectangular magnet with a mean field of $0.6 \mathrm{~T}$ over $5 \mathrm{~cm}$, placed on a translation stage inside the vacuum chamber, when introduced in the beam line, enables determination of the energy spectrum of the electron beam. The deflected electrons, in the presence of the magnet, impinge on a scintillating screen from Kodak (Lanex) which was then imaged by a 12-bit CCD camera placed outside the vacuum chamber. The transmitted laser light was prevented from reaching the Lanex screen by a thin aluminum foil, which was transparent to the electrons. Without the magnetic field the undeflected, electron beam passed through an $8 \mathrm{~mm}$ thick glass exit window that forms the boundary between the vacuum chamber and the room. After the electron beam exits the vacuum chamber it traverses a distance of up to several meters and impinges on a storage phosphor plate (Fuji MS-type) which is then read out using a Perkin Elmer CyclonePlus image-plate scanner, to infer the characteristics of the electron beam at the detector plane. Radiographic images were obtained by interposing various objects between the electron beam source and the phosphor plate.

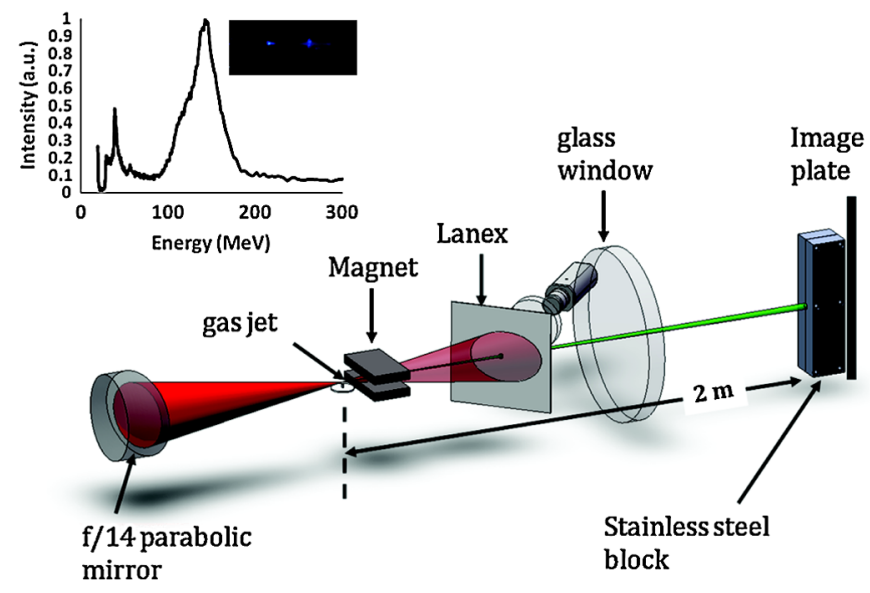

FIG. 1. Experimental schematic for electron beam generation. An f/14 off-axis parabolic mirror focuses a laser beam to a waist of $15 \mu \mathrm{m}$ onto a supersonic gas nozzle. High-energy electron beams generated in the laser-driven plasma are characterized using a magnetic spectrometer and then impinge on a fluorescent screen (Kodak-Lanex), which is imaged using a 12-bit CCD camera. Also depicted in the schematic is the experimental setup to record radiographs of embedded structures of submillimeter resolution at a large distance. The inset shows the dispersed electron beam and the corresponding energy spectrum after passage through the magnetic spectrometer. 


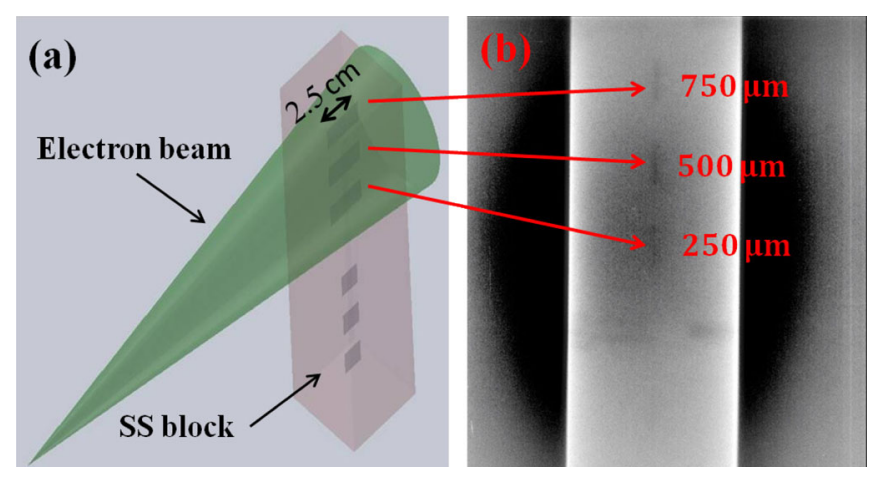

FIG. 2. (a) Three-dimensional representation of the stainless steel block with embedded voids. The electron beam used to image the voids is also shown schematically. (b) Experimental radiograph of the voids using electron beams with the characteristics shown in Fig. 1.

An important consideration for the electron beam used for the present study is its small source size and, hence, the anticipated viability in the detection of defects and voids in materials. In order to measure the resolving power of the electron beam, we constructed a stainless steel block of thickness $5 \mathrm{~cm}$ and width $5 \mathrm{~cm}$, with submillimeter gaps of width 250, 500 and $750 \mu \mathrm{m}$, embedded within it. The machined object consisted of $2.5-\mathrm{cm}$ long voids that were embedded within a 5-cm thick stainless steel block [shown in Fig. 2(a)], and placed 2-m from the supersonic jet. Three different void thicknesses were imaged, with widths of 250, 500, and $750 \mu \mathrm{m}$. Ten electron beam shots, each with electron energy $150 \pm 20 \mathrm{MeV}$ and an angular divergence of $4.7 \pm 0.5 \mathrm{mrad}$, were used to acquire the radiograph shown in Fig. 2(b), which was recorded by an image plate placed behind the steel block. The $250 \mu \mathrm{m}$ gap is better visually resolved as compared to the $750 \mu \mathrm{m}$ gap as the object was arranged in such a manner so as to center the electron beams over the $250 \mu \mathrm{m}$ void.

The images of the void can be used to determine the resolving power of the source. As a first step, in order to improve the signal-to-noise ratio on these images, Fourier filtering techniques were used. This involves the use of a bandpass filter which eliminated features smaller than 3 pixels and larger than 40 . The choice of the lower and upper cutoffs was based on the fact that the images of the voids being studied had a spatial size of 10-20 pixels. The result of applying this filter to the image in Fig. 2(b) is shown in Fig. 3(a). Figure 3(b) shows a lineout of the 500- $\mu \mathrm{m}$ gap taken at location 2, as well as a Gaussian fit to the data. To compute the spatial resolution obtained by this method, the derivative of this lineout across the $750-\mu \mathrm{m}$ gap (location 1 ) was computed, and is shown in Fig. 3(c). The full width at half maximum (FWHM) of the derivative yields a resolution of $330 \mu \mathrm{m}$. To the best of our knowledge, this is the highest resolution ever achieved for a radiographic image of an embedded void.
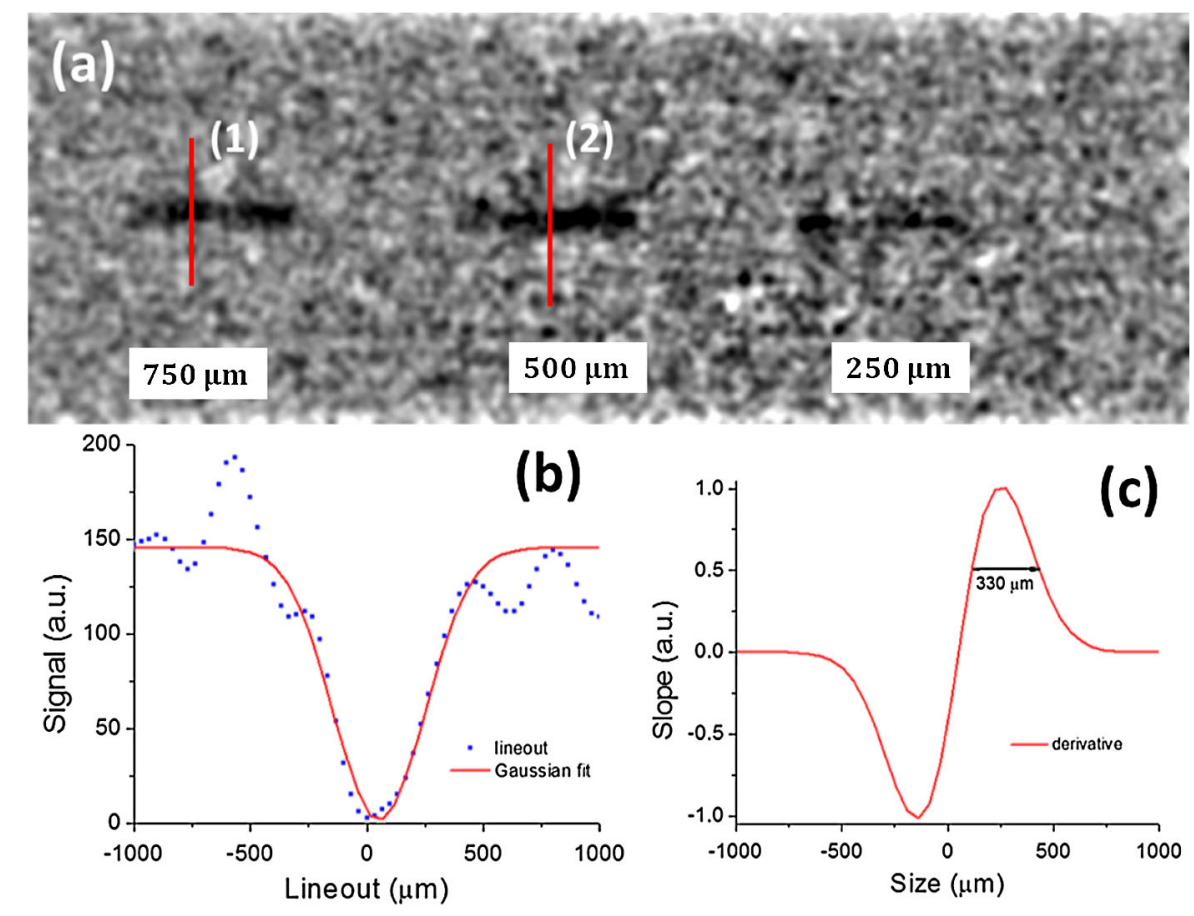

FIG. 3. Electron radiographic images of voids embedded in steel (from left to right: 750, 500, and 250 $\mu \mathrm{m}$ ). (a) Fourier enhanced image (red line indicates lineout location). (b) Lineout of crack (circles) and Gaussian fit (solid line). (c) Derivative of fitted profile indicates a source size of $330 \mu \mathrm{m}$. 
Since the source size of the electron beam is expected to be $<100 \mu \mathrm{m}$, the measured source size necessarily reflects the fact that the technique is limited by the size of the object being used. We computed the source size at different locations. As an example, a lineout taken at location 1 yields a source size of $410 \mu \mathrm{m}$. The calculations performed on the 500 and $750 \mu \mathrm{m}$ voids yield an average source size of $400 \pm 100 \mu \mathrm{m}$. The low contrast of the $250 \mu \mathrm{m}$ void, unfortunately, prevented its use in computing the source size. This, to the best of our knowledge, is the first evidence of submillimeter electron beam radiograph at a distance of 2-m from the source.

\section{RESULTS AND MONTE CARLO SIMULATIONS}

In order to predict the performance of the electron beam radiography device and compare this with measurements, the different experimental scenarios were modeled by means of Monte Carlo simulation code MCNPX v.2.5.0. Studies of the resolution possible with the laser-driven electron beam were numerically modeled assuming an electron beam emanating from a point source with a monochromatic energy of $150 \mathrm{MeV}$ and a divergence of $4.7 \mathrm{mrad}$ directed at the center of the $250 \mu \mathrm{m}$ gap. Both electrons and photons were tracked in MCNPX using the default physics models and approximately $3 \times 10^{7}$ source histories. In order to expedite the calculation, electron tracking in the system ceases when the electron energy falls below $100 \mathrm{keV}$. These low energy electrons are produced as the source electrons slow down in the target block. Geometric splitting was performed throughout the steel block to increase the electron population entering the image plate. The image-plate response was computed using an energy deposition mesh tally over its entire plane. The total energy deposited from all the particles in each mesh cell by all the particles per unit volume is included. The mesh consisted of a 200 by $200 \mu \mathrm{m}$ grid, averaged along the image-plate thickness. Figure 4, shows the experimental data and its comparison with the computed images. Figure 4(b) shows the results of the image-plate mesh tally in units of $\mathrm{MeV}$ per source particle per $\mathrm{cm}^{3}$. The 750, 500, and $250 \mu \mathrm{m}$ wide, $2.5-\mathrm{cm}$ long microcracks are visible at approximate positions of $-8,-5$, and $-2.5 \mathrm{~cm}$ in good visual agreement with the experimental radiograph in Fig. 4(a). The electron beam source size is theoretically predicted to be the same size as the spot size of the driving laser beam [15].

While it was impractical for us to experimentally conduct truly long-standoff studies (at distance of 10-100 m from the source), MCNPX simulations provided a reliable way to extend the results. A Monte Carlo model was developed to investigate the propagation of high-energy electron beams in air over significant distances. As an example of these calculations, we show in Fig. 5 the propagation of a monoenergetic source of electron beam of energy $400 \mathrm{MeV}$. The choice of beam parameters is determined by experimentally measured characteristics. The beam travels a distance of $100 \mathrm{~m}$ from the source, in air to reach a tally plane. The computed electron fluence (normalized to maximum) of the resulting shower is also shown in Fig. 5. The electron beam spreads to a diameter of $1 \mathrm{~m}, \sim 20 \%$ higher than would be expected from geometrical considerations alone. The result indicates that a significant fraction of the electrons undergo little or no deflection through a distance of $100 \mathrm{~m}$ of air making it a good candidate for applications where the object cannot be placed in close proximity to the source. Also, electron beams, unlike $\mathrm{MeV}$ photons, can be manipulated, redirected, or focused either before, during, and after interaction with the target. The signal-to-noise level can be improved by means of magnetic filtering of secondary electrons.

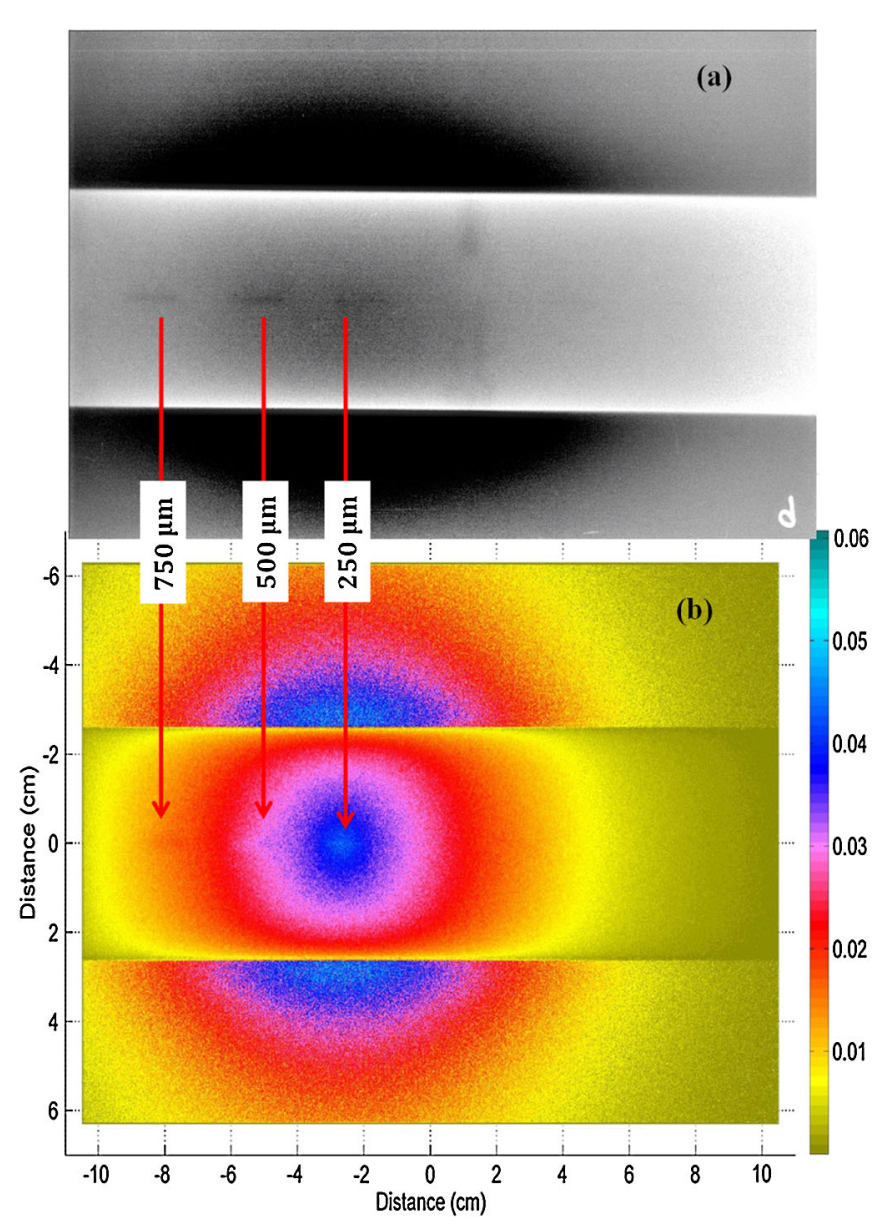

FIG. 4. (a) Processed experimental radiograph indicating the embedded gaps of width 750, 500, and $250 \mu \mathrm{m}$. (b) Monte Carlo simulated radiograph shows reasonable agreement with the experimentally obtained image where the 750, 500, and $250 \mu \mathrm{m}$ voids are visible at $-8,-5$, and $-2.5 \mathrm{~cm}$, respectively. The intensity numbers on the color bar code indicate the energy deposited by the electrons on the image plate, in $\mathrm{MeV}$ per source electron in each voxel. The voxel volume considered in the MC simulation is $200 \mu \mathrm{m} \times 200 \mu \mathrm{m} \times 115 \mu \mathrm{m}$. 


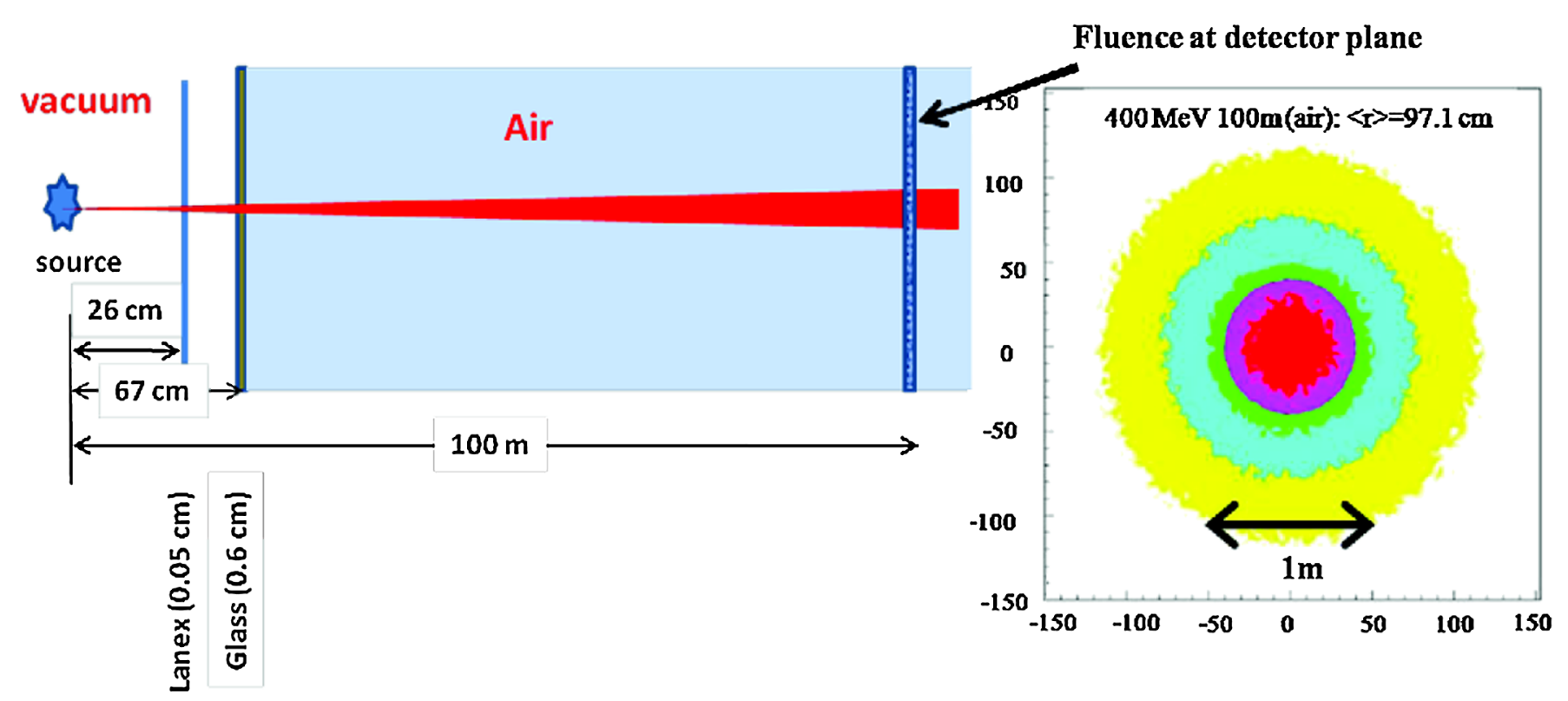

FIG. 5. A Monte Carlo simulation of a point source electron beam of energy $400 \mathrm{MeV}$ with a cone angle of 8 mrad, exiting the vacuum chamber to reach a tally plane $100 \mathrm{~m}$ from the source. The electron beam spreads to a diameter of about a meter at this distance whereas the unscattered beam would measure $0.8 \mathrm{~m}$ in diameter, at the same distance from the source.

\section{CONCLUSIONS}

In summary, we have demonstrated radiography of complex structures embedded in dense material using highenergy laser-accelerated electron beams. This research addresses a pressing need for reliable nondestructive techniques to identify the presence of defects in dense structures, ranging from turbine blades to nuclear reactor vessels. The submillimeter resolution demonstrated by this technique will permit the study of the growth of microstructures as critical materials age. To our knowledge, this is the first demonstration of an electron beam radiographic technique capable of resolving submillimeter embedded features in dense materials at a large distance from the source. Coupled with the simplicity and low cost of the detection system, our results provide a compelling case for further development of laser-driven electron radiography systems for nondestructive studies and detection at long standoff.

\section{ACKNOWLEDGMENTS}

This work is funded by the Air Force Office of Scientific Research (AFOSR), the Defense Advanced Research Projects Agency (DARPA), the Chemical Sciences, Geosciences and Biosciences Division, Office of Basic Energy Sciences, Office of Science, U.S. Department of Energy (DOE), and the Domestic Nuclear Detection Office (DNDO) of the Department of Homeland Security (DHS). We thank Jeff Thomas for providing the three-dimensional drawing.
[1] T. Tajima and J.M. Dawson, Phys. Rev. Lett. 43, 267 (1979).

[2] V. Malka, J. Faure, Y. A. Gaudel, E. Lefebvre, A. Rousse, and K. A. Phuoc, Nature Phys. 4, 447 (2008).

[3] S. Fritzler, E. Lefebvre, V. Malka, F. Burgy, A. E. Dangor, K. Krushelnick, S.P.D. Mangles, Z. Najmudin, J.-P. Rousseau, and B. Walton, Phys. Rev. Lett. 92, 165006 (2004).

[4] J. Faure, Y. Glinec, A. Pukhov, S. Kiselev, S. Gordienko, E. Lefebvre, J.-P. Rousseau, F. Burgy, and V. Malka, Nature (London) 431, 541 (2004).

[5] C. G. R. Geddes, C. Toth, J. van Tilborg, E. Esarey, C. B. Schroeder, D. Bruhwiler, C. Nieter, J. Cary, and W.P. Leemans, Nature (London) 431, 538 (2004).

[6] S.P.D. Mangles, C.D. Murphy, Z. Najmudin, A. G. R. Thomas, J. L. Collier, A.E. Dangor, E. J. Divall, P.S. Foster, J. G. Gallacher, C. J. Hooker, D. A. Jaroszynski, A. J. Langley, W. B. Mori, P. A. Norreys, F. S. Tsung, R. Viskup, B.R. Walton, and K. Krushelnick, Nature (London) 431, 535 (2004).

[7] N. Cunningham, S. Banerjee, V. Ramanathan, N. Powers, N. C-Smith, R. Vane, D. Schultz, S. Pozzi, S. Clarke, J. Beene, and D. Umstadter, in Proceeding of the 20th International Conference on the Application of Accelerators in Eesearch and Industry [Nucl. Instrum. Methods Phys. Res., Sect. B (to be published)].

[8] S. Banerjee, N. Powers, V. Ramanathan, B. Shadwick, and D. Umstadter, in Proceedings of the 23rd Particle Accelerator Conference [Phys. Rev. ST Accel. Beams (to be published)].

[9] J. L. Jones, D. R. Norman, K. J. Haskell, J. W. Sterbentz, W. Y. Yoon, S. M. Watson, J. T. Johnson, J. M. Zabriskie, B. D. Bennett, R.W. Watson, C.E. Moss, and J.F. 
Harmon, Nucl. Instrum. Methods Phys. Res., Sect. A 562, 1085 (2006).

[10] J. L. Jones, B. W. Blackburn, S. M. Watson, D. R. Norman, and A. W. Hunt, Nucl. Instrum. Methods Phys. Res., Sect. A 261, 326 (2007).

[11] NIST stopping power and range tables for electrons [http://physics.nist.gov/PhysRefData/Star/Text/ ESTAR.html].

[12] N. S. P. King, E. Ables, Ken Adams, K. R. Alrick, J.F. Amann, Stephen Balzar, P.D. Barnes, Jr., M.L. Crow, S. B. Cushing, J. C. Eddleman, T. T. Fife, Paul Flores, D. Fujino, R. A. Gallegos, N. T. Gray, E. P. Hartouni, G. E. Hogan, V.H. Holmes, S. A. Jaramillo, J. N. Knudsson, R. K. London, R. R. Lopez, T.E. McDonald, J.B. McClelland, F.E. Merrill, K. B. Morley, C. L. Morris,
F. J. Naivar, E. L. Parker, H. S. Park, P. D. Pazuchanics, C. Pillai, C. M. Riedel, J. S. Sarracino, F. E. Shelley, Jr., H. L. Stacy, B.E. Takala, Richard Thompson, H.E. Tucker, G. J. Yates, H. J. Ziock, and J. D. Zumbro, Nucl. Instrum. Methods Phys. Res., Sect. A 424, 84 (1999).

[13] S. P. D. Mangles, B. R. Walton, Z. Najmudin, A.E. Dangor, K. Krushelnick, V. Malka, M. Manclossi, N. Lopes, C. Carias, G. Mendes, and F. Dorchies, Laser Part. Beams 24, 185 (2006).

[14] Y. Glinec, J. Faure, L. Le Dain, S. Darbon, T. Hosokai, J. J. Santos, E. Lefebvre, J. P. Rousseau, F. Burgy, B. Mercier, and V. Malka, Phys. Rev. Lett. 94, 025003 (2005).

[15] F. V. Hartemann, D. J. Gibson, W. J. Brown, A. Rousse, K. T. Phuoc, V. Malka, J. Faure, and A. Phukov, Phys. Rev. ST Accel. Beams 10, 011301 (2007). 